\title{
Burden in Schizophrenia: A Literature review
}

\author{
Hanchun Yang ${ }^{1, a,{ }^{*}, \dagger}$ Hanyang $\mathrm{Yu}^{2, b, *}, \dagger$ \\ ${ }^{I}$ The College of Liberal Arts and Sciences , Arizona State University , Tempe , Arizona State , United States of Amer- \\ ica \\ ${ }^{2}$ University of Connecticut, Storrs, Connecticut, United States of America

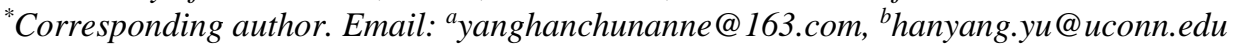

${ }^{\dagger}$ These authors contributed equally.

\begin{abstract}
Although some researchers have made studies on some aspects of the burden of schizophrenia patients, there is not a comprehensive focus. This paper will focus on both the hard burden (easily quantifiable data, such as economic) and the soft burden (difficult to quantify data) of schizophrenia patients. Additionally, data on the economic burden of schizophrenia vary across countries or regions due to differences in mental health service delivery, economic development, and health care coverage systems. Mental illness is much less understood in general in developing countries, and patients receive less assistance. Therefore, this paper aims to summarize the burden of schizophrenia by examining data from developing countries. This paper assessed the disease burden of schizophrenia through a literature review. The data searched were largely international databases such as ScienceDirect. The search found s studies covering four aspects of the burden: economic, family, physical, and social. It was concluded that the burden suffered by people with schizophrenia in developing countries is substantial. The disease imposes a heavy economic, psychological, and physical burden on patients in developing countries. In addition, the family burden and social burden caused by schizophrenia should be given full public attention.
\end{abstract}

Keywords: Schizophrenia, Hard burden, Soft burden

\section{INTRODUCTION}

Schizophrenia is a chronic, prolonged psychiatric disorder with a high recurrence rate and high disability rate, mostly in young adults. According to a 2004 survey by the World Health Organization (WHO), schizophrenia is among the 20 leading causes of disability. 26.3 million people are currently suffering from schizophrenia [1]. Not only does this disorder have a considerable impact on the mental health of patients, but they also experience physical, economic, and social interaction barriers, and it can affect their surroundings. Previous studies indicated that the burden of psychological reactions, family-related stressors, economic and social which arise from schizophrenia would also in no way be ignored [2]. However, most of the research on schizophrenia-related burden was conducted in western cultural background developed countries. There are few studies focus on the schizophrenia-related burden in developing countries.

The present review aims to summarize previous studies on the schizophrenia-related burden in the context of domestic and international research. In particular, it highlights that the burden associated with schizophrenia in the developing world is a matter of urgent concern.

\section{METHODS}

To identify all relevant literature reviews on the different dimensions of the burden of schizophrenia in developing countries, we searched for systematic literature reviews. Most of the literature found through the school library's search engine as an entry point was from ScienceDirect. Still, since this paper focuses on data from developing countries, the information from international repositories did not fully meet our needs. We also adopted some of the literature from national journals. This part of the literature was rigorously selected, and the experimental data were obtained by means of highly generalized measurements and calculations. 


\section{RESULTS}

\subsection{Physiology Burden}

Schizophrenia has long been proved to positively associate with mortality rate and a negative association with average life span. According to Mortensen and Juel, schizophrenia reduces a schizophrenia patient's average life span by 10 years. Moreover, the risk of male schizophrenia patients' mortality was increased by 4.7 fold, and the risk of female schizophrenia patients' mortality was increased by 2.3 fold [3]. Multiple factors may increase a schizophrenia patient's mortality rate, including suicide action, non-traditional cardiovascular disease risk factors [4], systemic inflammation, and reduced cognitive abilities.

Indeed, long-duration treatment of antipsychotic drugs could statistically increase a schizophrenia patient's life span. However, antipsychotic drugs also have unavoidable side effects which may affect a patient's quality of life [5]. Antagonism of D2 receptors, a type of 1st generation of antipsychotic drugs, is associated with an increased risk of extrapyramidal symptoms. As for 2 nd generation of antipsychotic drugs, it cannot be denied that their neurological side effects are lower, but they do have relatively higher metabolic side effect risks. According to M. Alvarez-Jiménez et al.'s research [6, 7], 86\% of schizophrenia patients who took Quetiapine gained more than $75 \%$ body weight from baseline.

\subsection{Family/Caregivers Burden}

In recent years, the rapid development of community mental health services has led to the return of many formerly long-term hospitalized patients to their families. According to Goldman [8], about $65 \%$ of these patients are cared for by their family members. After years of practice, many psychiatrists and social workers have recognized that the family is an important component of the community mental health service system. The quality of community mental health services depends largely on the attitude of the family members and their ability to care for the patients. Still, at the same time, the re-entry of these patients into the family inevitably impacts the family and creates a number of related problems.

In this article, family burden refers to the problems, difficulties, or adverse effects that a person with schizophrenia has on his or her family and related members, caregivers. The patient's illness symptoms and behaviors become a disruptive factor in family life and the resulting events, which will be mentioned below, regarding physical, social, family relationships, and mental stress.

In Ethiopia, as in most developing countries, there are not enough services to help people with mental disorders; therefore, the family is often the sole caregiver for relatives of people with serious mental illness. According to the study [9], female relatives experienced more difficulty going to work or stopped working due to the need to care for a relative with schizophrenia. However, there was no difference between male and female spouses in terms of work burden. As spouses of schizophrenia cases, there was a significant association between caring for symptomatic disorders and work burden in all cases [9]. Caring for someone with schizophrenia can be a demanding and exhausting activity. It challenges the physical and emotional resources of family caregivers. In traditional societies like India, this situation is exacerbated by illiteracy. In Stanley, Balakrishnan, and Ilangovan's experiment[10], patient family members' perceived burden was assessed using The Zarit Burden Interview (ZBI). Scores above 60 were categorized as "severe burden," resulting in all caregivers in the experiment being judged as severely burdened. Also, in this experiment, according to The Schizophrenia Caregiver Quality of Life questionnaire, the majority of people were classified as "low" based on the median, on the total quality of life score, as well as on all seven dimensions of the S-CGQoL. As measured by The Depression, Anxiety and Stress Scale (DASS-21) [10], while the majority of caregivers were experiencing "moderate" levels of stress in terms of depression and anxiety, some were classified as "severe" and "extremely severe" [10]. Salleh and his companion's research in Malaysia showed that $23 \%$ of caregivers who were stressed suffered from neurotic disorders and 50\% showed symptoms of neurotic depression) [11]. A study in China showed that more than $60 \%$ of people believed that discrimination caused great psychological stress to family members and seriously affected the daily life of patients and family members, especially social interactions [12].

\subsection{Economy Burden}

As a psychotic disorder that requires considerable time and effort during the antipsychotic treatment procedure, schizophrenia's economic burden could be almost unbearable to the patients who live in developing countries. Due to developing countries' problematic public health care system, low productivity, lack of education, and social stigma [13], both direct and indirect cost of schizophrenia has largely increased.

Direct costs, which are directly related to the disease, include 1. direct medical costs: medical services for diagnosis, treatment, medication, care, rehabilitation, counseling, etc. 2. direct non-medical services: the costs of other resources related to the disease, such as transportation to the clinic, accommodation, etc.

Indirect costs include three main components: 1. the cost of damage to the patient's resources caused by the disease, that is, the cost of property damage 2 . the economic loss due to the loss of work related to the disease 3 . the reduction in life expectancy due to the death of the patient due to the disease, which is calculated according to the 
human capital approach, taking into account the patient's gender, age, and life expectancy.

299 Chinese patients who were diagnosed with schizophrenia participated in Zhai et al.'s study. They completed an Economical Cost Questionnaire every two months for a year. According to Zhai et al.'s data [14], "The median annual costs in Chinese currency per patient were $¥ 4370$ (approximately US \$700) in direct costs and $¥ 10,081$ (approximately US \$1600) in indirect costs. Direct costs for those patients who had at least one hospitalization (¥7614) were substantially higher than those who were not hospitalized (¥1812; P, 0.001). Caregivers bore a substantial part of the indirect costs (¥3600). Have to notice that the average net income of a rural resident was only $¥ 2936$ in 2004, China.

Also, the lack of affordable psychotic treatment options in less-developed regions only makes things worse. Phillips MR et al.'s previous epidemic study [15] in China shows that $27.6 \%$ of psychotic disorder patients never sought any proper treatment. Besides economic \& educational factors, the very limited number of mental health care institutions is also a major reason. According to WHO's data [16], the psychiatric bed ratio per 10,000 population in China was 1.06 in 2005, compared with 8.00 in Europe and 7.70 in the US.

\subsection{Society Burden}

Because of the inadequate welfare support and advocacy policies for people with mental illness in developing countries, people with mental illness in developing countries are more hindered in their social activities. In addition, because of the low awareness of the mental illness, patients are more limited in their social activities and suffer more psychological stress. These are the social barriers that patients suffer from, as defined in this paper.

First, in patients with social burden, stress can be measured by their resistance to social interaction, also known as social anxiety. The results of a study in China showed that $42 \%$ of patients reported that they were treated unfairly by their units, discriminated against by colleagues or classmates, looked down upon by neighbors, and even led to failed relationships or marriages [17]. Due to the lack of prevalence and popular awareness, the unfair treatment of mentally ill patients in society is very common in developing countries. This paper will explore the unfair treatment of schizophrenic patients in society and some of their inconvenient situations.

According to researchers, patients with schizophrenia living in the community may still experience burdens imposed by their surroundings even after recovering from unpleasant psychiatric symptoms. These patients in remission who live in the community have been found to develop or worsen symptoms of social anxiety [18]. The reason for classifying this point as a social disorder in patients is that social anxiety symptoms can negatively affect their social functioning or subjective quality of life. A study by Indian scholar Kumazaki et al. [19] was conducted to demonstrate this point. Since the topic of this paper is to demonstrate that patients with schizophrenia in developing countries suffer from a greater impairment, data from experiments that can discuss the severity of social anxiety symptoms in these patients are cited. In this study, experimenters used the Liebowitz Social Anxiety Scale (LSAS) to measure the severity of social anxiety symptoms in patients with schizophrenia. At year 5 follow-up, 24 patients $(67 \%)$ had a mean LSAS total score above 30, indicating their social anxiety symptoms had reached a clinical level. In addition, 2 patients $(5.5 \%)$ had a "very severe" level of symptoms (LSAS total score $\geq 95$ ), 2 patients (5.5\%) had "severe" symptoms (LSAS total score <80), and 6 patients $(17 \%)$ had "marked" symptoms (LSAS total score <65). LSAS average total score deteriorates over time [20].

A very important influencing factor in the social rehabilitation of schizophrenic patients is social support. However, in developing countries, due to low social and general awareness of schizophrenia, the government and society provide less welfare and support. Patients do not have a good perception of the support provided by society. In a cross-sectional study of 160 patients with schizophrenia receiving community psychiatric services at Hospital Kuala Lumpur [20] (HKL), the experimenter used the Multidimensional Scale of Perceived Social Support (MSPSS) to assess the level of social support felt by the patients. About $72 \%$ of respondents had poor perceived social support, with the lowest level of support coming from significant others, followed by friends and family [20].

In most developed countries, their welfare systems provide a "safety net" for the masses, so the burden of unemployment can be shared by the government. In contrast, without a welfare system that protects individuals who cannot work due to mental illness, workers in developing countries are more likely to continue working while disabled. This leads to a greater probability that individuals with mental illness in developing countries will suffer from employment difficulties and stress in the workplace. Unfortunately, no English-language research literature was found that could be referenced on the relationship between employment rates and mental illness in developing countries. However, it is reasonable to assume that the employment status of people with mental illness in developing countries will only be worse than that of people in developed countries due to a more abundant labor force and the absence of sound policy protections. One U.S. study found that employment rates decreased with increasing severity of mental illness (no mental illness, 75.9\%; mild, $68.8 \%$; moderate, $62.7 \%$; severe, $54.5 \%$, p<.001). More than one-third of persons with severe mental illness had incomes below $\$ 10,000$ [21]. 
In addition to employment difficulties, the working environment in developing countries is also more unfriendly to the mental state of people with mental illness and normal people, according to the study. According to the study, employers in developing countries may be more inclined to force employees to attend work when they are not feeling well. Therefore a higher incidence of mental illness can be expected to be associated with this [22]. In searching the literature, it was found that most workplace mental health research has been conducted in developed countries, and it can be argued that there are gaps in research in this area in all developing countries. The lack of emphasis on the impact of the work environment on mental health in developing countries further suggests that the work environment in developing countries is less friendly to workers' mental health. Of course, this is only a hypothesis to draw the attention of researchers in developing countries due to the lack of experimental data to support it.

\section{DISCUSSION}

This review, to inform governments in developing countries, focuses on data from developing countries. However, because of the slight limitations of research in this area in developing countries, the burden of the considered, especially the physiological burden, may also be discussed more extensively with reference to research in developed countries. By means of the comprehensive description of the 28 articles, it was compiled that people with schizophrenia in developing countries have proven to endure significant barriers in many ways. Schizophrenia is a clinically common severe mental disorder. The burden of illness it causes is enormous, exceeding that of many other mental disorders, especially the economic burden and family burden, and should be given great attention.

Due to the limitations of the reference literature, this review could not quantify the impact of schizophrenia on family functioning and social functioning with the same criteria. Magliano et al. [23] used the Family Problems Questionnaire (FPQ) and the Social Network Questionnaire (SNQ) to examine the Italian population. Questionnaire (FPQ) and social network questionnaire (SNQ) in a series of 709 schizophrenic caregivers and 646 caregivers of other psychiatric patients in 30 study centers in Italy found significant social activity limitations and negative effects on family life in both groups, with poorer social support and higher subjective burden in schizophrenic caregivers. It is highly desirable to standardize the criteria and quantify more clearly the family and social burden of schizophrenia patients. Although this review did not find studies in developing countries that met such criteria, we cited additional, different, and more refined factors to demonstrate that people with schizophrenia experience high burden levels.

The situation could be extremely different for schizophrenia patients in developed and developing countries.
For developing countries' patients, not only do they have to face physiological burdens which threaten their physical wellbeing, but they also have to face economic \& social burdens which some of them are exclusive to less developed regions. Over the last decades, the number of schizophrenia patients was increasing steadily.

Since schizophrenia has become an important public health problem, the barriers encountered by people with schizophrenia are more pronounced in developing countries. The government should play a key role in reducing the physical, economic, family, and social burden of schizophrenia by improving the risk-sharing mechanism of the disease burden and facilitating patients' access to systematic antipsychotic treatment and functional rehabilitation through adequate medical coverage and social security. Most developing countries have a large patient population base, low treatment rates, and a shortage of mental health resources. They face greater challenges in improving mental health service systems and reducing the burden of illness. Mental health professionals should pay attention to the results of academic research, and appropriately base on available research data to advance the development of mental health services, and conduct countryspecific research on mental health practice to provide a basis for developing and improving relevant policies.

\section{CONCLUSION}

This review highlights the burden of schizophrenia in developing countries, including biological, family, economic and social aspects. It is important to provide supportive interventions for patients and their families to reduce the burden of schizophrenia.

\section{REFERENCES}

[1] Mathers, C. (2008). The global burden of disease: 2004 update. World Health Organization.

[2] Shibre, et al. Schizophrenia: Illness impact on family members in a traditional society - Rural ethiopia, 2003. Social Psychiatry and Psychiatric Epidemiology $38(1): 27-34$

[3] Mortensen, P. B., \& Juel, K. (1993). Mortality and Causes of Death in First Admitted Schizophrenic Patients. British Journal of Psychiatry, 163(2), 183-189.

[4] Chung, K., Chen, P., Kuo, C., Tsai, S., Huang, S., \& Wu, W. (2018). Risk factors for early circulatory mortality in patients with schizophrenia. Psychiatry Research, 267, 7-11.

[5] Roldán, A., Galling, B., \& Correll, C. (2016). Antipsychotic co-initiation versus monotherapy in schizophrenia: a systematic review, meta-analysis and metaregression analysis. European Neuropsychopharmacology, 26(S2), 537-538. 
[6] M. Alvarez-Jiménez, C. González-Blanch, J.L. Vázquez-Barquero, R. Pérez-Iglesias, O. MartínezGarcía, T. Pérez-Pardal, et al.Attenuation of antipsychotic-induced weight gain with early behavioral intervention in drug-naive first-episode psychosis patients: a randomized controlled trial, J Clin Psychiatry, 67 (8) (2006), pp. 1253-1260

[7] Huhtaniska, S., Jääskeläinen, E., Heikka, T., Moilanen, J. S., Lehtiniemi, H., Tohka, J., . . . Miettunen, J. (2017). Long-term antipsychotic and benzodiazepine use and brain volume changes in schizophrenia: The Northern Finland Birth Cohort 1966 study. Psychiatry Research: Neuroimaging, 266, 73-82.

[8] Goldman, H. H. (1982). Mental illness and family burden: A public health perspective. Hospital \& Community Psychiatry, 33(7), 557-560.

[9] Shibre, T., Kebede, D., Alem, A., Negash, A., Deyassa, N., Fekadu, A., ... \& Kullgren, G. (2003). Schizophrenia: illness impact on family members in a traditional society-rural Ethiopia. Social Psychiatry and Psychiatric Epidemiology, 38(1), 27-34.

[10] Stanley, S., Balakrishnan, S. \& Ilangovan, S. (2017) Psychological distress, perceived burden and quality of life in caregivers of persons with schizophrenia, Journal of Mental Health, 26(2): 134-141.

[11] Salleh, M. R. (1994). The burden of care of schizophrenia in Malay families. Acta Psychiatrica Scandinavica, 89(3), 180-185.

[12] Phillips, M. R., Yang, G., Li, S., \& Li, Y. (2004). Suicide and the unique prevalence pattern of schizophrenia in mainland China: a retrospective observational study. The Lancet, 364(9439), 1062-1068.

[13] Montgomery, W., Liu, L., Stensland, M. D., Xue, H. B., Treuer, T., \& Ascher-Svanum, H. (2013). The personal, societal, and economic burden of schizophrenia in the People's Republic of China: implications for antipsychotic therapy. ClinicoEconomics and outcomes research: CEOR, 5, 407.

[14] Zhai JG, Zhao JP, Chen JD. An investigation on economic costs of schizophrenia in two medical establishments in Hunan and Shandong. Chinese Journal of Psychiatry. 2007;40(1):41-44.

[15] Phillips, M. R., Zhang, J., Shi, Q., Song, Z., Ding, Z., Pang, S., ... \& Wang, Z. (2009). Prevalence, treatment, and associated disability of mental disorders in four provinces in China during 2001-05: an epidemiological survey. The Lancet, 373(9680), 2041-2053.

[16] World Health Organization. Mental health atlas 2005 [webpage on the Internet]. Geneva: World Health Organization; 2005. Available from: http://www.who.int/mental_health/evidence/mhatlas05/en/.

[17] Liu Yufang, Song Changxue, Yu Xiumei, et al. Epidemiological survey of mental disorders in Huairou District, Beijing. Journal of Clinical Psychosomatic Disorders, 2005, 11 (1): 23 - 26.

[18] Mizuno, Masafumi; Sakuma, Kei; Ryu, Yonosuke Munakata,Shunichi;Takebayashi, Toru ; Murakami, Masaaki ; Falloon, Ian R.H ; Kashima, Haruo Japan: The Keio Journal of Medicine Keio journal of medicine, 2005, Vol.54 (2), p.95-101

[19] Kumazaki H, Kobayashi H, Niimura H, Kobayashi Y, Ito S, Nemoto T, Sakuma K, Kashima H, Mizuno M. Lower subjective quality of life and the development of social anxiety symptoms after the discharge of elderly patients with remitted schizophrenia: a 5-year longitudinal study. Compr Psychiatry. 2012 Oct;53(7):946-51.

[20] Munikanan T, Midin M, Daud TIM, Rahim RA, Bakar AKA, Jaafar NRN, Sidi H, Baharuddin N. Association of social support and quality of life among people with schizophrenia receiving community psychiatric service: A cross-sectional study. Compr Psychiatry. 2017 May;75:94-102.

[21] Luciano, A., \& Meara, E. (2014). Employment status of people with mental illness: national survey data from 2009 and 2010. Psychiatric Services, 65(10), 1201-1209.

[22] Chopra, P. (2009). Mental health and the workplace: issues for developing countries. International Journal of Mental Health Systems, 3(1), 1-9.

[23] Magliano, L., Fiorillo, A., Malangone, C., De Rosa, C., \& Maj, M. (2006). A memorial tribute: Patient functioning and family burden in a controlled, realworld trial of family psychoeducation for schizophrenia. Psychiatric services, 57(12), 1784-1791. 